\title{
Effects of Cigarette Smoking and Its Cessation on Lipid Metabolism and Energy Expenditure in Heavy Smokers
}

\author{
Marc K. Hellerstein, *\$ Neil L. Benowitz, ${ }^{\star}$ Richard A. Neese, " 3 Jean-Marc Schwartz, \\ James Hsieh, and Dennis Faix \\ Divisions of * Endocrinology and Metabolism, and ${ }^{\ddagger}$ Clinical Pharmacology, Department of Medicine, San Francisco General Hospital, \\ University of California, San Francisco, California 94110; and ${ }^{\S}$ Department of Nutritional Sciences, University of California at Berkeley, \\ Berkeley, California 94720
}

\begin{abstract}
The relationship between thermogenic and potentially atherogenic effects of cigarette smoking (CS) and its cessation was investigated. Heavy smokers $(n=7$, serum cotinine $>200 \mathrm{ng} /$ $\mathrm{ml},>20$ cigarettes $/ \mathrm{d}$ ) were maintained on isoenergetic, constant diets for 2 wk, 1 wk with and 1 wk without CS. Stable isotope infusions with indirect calorimetry were performed on day 7 of each phase, after an overnight fast. CS after overnight abstention increased resting energy expenditure by $5 \%$ (not significant vs. non-CS phase; $P=0.18$ ). CS increased the flux of FFA by $77 \%$, flux of glycerol by $82 \%$, and serum FFA concentrations by $73 \%$ ( $P<0.02$ for each), but did not significantly affect fat oxidation. Hepatic reesterification of FFA increased more than threefold $(P<0.03)$ and adipocyte recycling increased nonsignificantly $(P=0.10)$. CS-induced lipid substrate cycles represented only $15 \%$ (estimated $11 \mathrm{kcal} / \mathrm{d}$ ) of observed changes in energy expenditure. De novo hepatic lipogenesis was low ( $<1-2 \mathrm{~g} / \mathrm{d})$ and unaffected by either acute CS or its chronic cessation. Hepatic glucose production was not affected by CS, despite increased serum glycerol and FFA fluxes. Cessation of CS caused no rebound effects on basal metabolic fluxes. In conclusion, a metabolic mechanism for the atherogenic effects of CS on serum lipids (increased hepatic reesterification of FFA) has been documented. Increased entry of FFA accounts for CS-induced increases in serum FFA concentrations. The thermogenic effect of $\mathrm{CS}$ is small or absent in heavy smokers while the potentially atherogenic effect is maintained, and cessation of CS does not induce a rebound lipogenic milieu that specifically favors accrual of body fat in the absence of increased food intake. (J. Clin. Invest. 1994. 93:265-272). Key words: lipolysis • substrate cycles • calorigenesis • atherogenesis • stable isotopes
\end{abstract}

\section{Introduction}

Cigarette smoking $(\mathrm{CS})^{1}$ is associated with reduced body weight and cessation of CS results in weight gain (1-11). The public is aware of this effect of CS, and concern about weight

Address correspondence to Dr. Marc K. Hellerstein, Division of Endocrinology and Metabolism, Department of Medicine, San Francisco General Hospital, 1001 Potrero Avenue, San Francisco, CA 94110.

Received for publication 12 April 1993 and in revised form 3 September 1993.

J. Clin. Invest.

(C) The American Society for Clinical Investigation, Inc.

$0021-9738 / 94 / 01 / 0265 / 08 \$ 2.00$

Volume 93, January 1994, 265-272 gain is one of the most common reasons given for continuing or returning to $\mathrm{CS}(1-4)$. Concern about weight gain after quitting smoking and the use of CS to control body weight is particularly prevalent in women smokers (3), who represent the fastest growing population of new smokers. Although the adverse health effects of CS greatly outweigh the benefits of lower body weight $(10,12)$, cosmetic or other factors clearly come into play.

The mechanism by which CS reduces body weight remains uncertain. Contrary to the general belief that CS reduces food intake, most published studies have reported increased food intake in smokers, in the range of $350 \mathrm{kcal} / \mathrm{d}(13,14)$, although others have reported reduced food intake in smokers (15-17). In experimental animals, chronic administration of nicotine or exposure to $\mathrm{CS}$ increases oxygen consumption and results in weight loss (10) and rats given nicotine lose weight without significant reduction in food intake (18). Recent metabolic ward studies confirm that CS has an acute calorigenic effect in humans. Smoking increases 24-h energy expenditure (EE) by $200 \mathrm{kcal} / \mathrm{d}$ (19) and intranasal or intravenous nicotine increases resting $\mathrm{EE}$ (REE) by $5-10 \%(20,21)$, although we (22) have recently observed a lower CS-induced increase in REE in heavy smokers compared with light smokers.

The metabolic basis of CS-induced calorigenesis is not understood. Nicotine causes activation of the sympathetic nervous system and the release of catechols $(23,24)$, which increase EE in humans $(25,26)$. CS or catechol administration increases serum FFA concentrations (27-29). One hypothesis has been that lipolysis or elevations in serum FFA concentrations contribute to the thermogenesis of CS (26). Consistent with this suggestion, norepinephrine-induced thermogenesis has been reported to be partially prevented by niacin (25), a lipolysis inhibitor, in humans. A precedent exists for lipolysis/ reesterification substrate cycling contributing to thermogenesis in a hyper-adrenergic clinical condition, body burns (30). FFA themselves are also known to increase cardiac oxygen consumption (31).

Potentially linked but undesirable consequences of nicotine-induced lipolysis are the increased triglyceride and LDL cholesterol concentrations and reduced HDL cholesterol concentrations associated with CS $(32,33)$. There is a dose-response relationship between the number of cigarettes smoked per day and serum cholesterol concentrations (34), and cessation of CS increases HDL cholesterol (33). In several dyslipi-

1. Abbreviations used in this paper: CS, cigarette smoking; DNL, de novo hepatic lipogenesis; EE, energy expenditure; FAME, fatty acidmethyl esters; GC, gas chromatography; LPL, lipoprotein lipase; M.E., molar excess; NPRQ, non-protein respiratory quotient; Ra, rate of appearance; REE, resting energy expenditure; TG, triglyceride. 
demic conditions, including diabetes (35-37), increased delivery of FFA to the liver and hepatic reesterification has been proposed to be the cause of increased VLDL triglyceride secretion and secondary alterations in serum lipids. Conversely, the mechanism by which niacin improves lipids in humans is believed to be through inhibition of lipolysis $(38-40)$. Hepatic reesterification of FFA may therefore link the thermogenic and atherogenic effects of CS. The effects of CS on lipolysis, serum FFA flux, and hepatic reesterification of FFA have not been determined in humans, however, nor have their relationships to CS-induced calorigenesis been evaluated.

With regard to cessation of CS, the suggestion has been made $(41,42)$ that rebound metabolic effects occur after smoking cessation that specifically favor the deposition of body fat and could therefore provide a metabolic driving force for increased adiposity (obesity) after smoking cessation. This proposal has not been tested from a quantitative perspective, however, by measuring fat synthesis and fat oxidation under conditions of constant dietary fat and energy intake.

Our objectives in this study were to evaluate relationships between lipolytic, calorigenic, and dyslipidemic effects of CS and determine the metabolic effects of cessation of CS. Four questions were addressed. (a) What are the effects of CS on lipid metabolism, carbohydrate metabolism, and REE in heavy smokers? $(b)$ Do substrate cycles account for the calorigenic effect of CS? $(c)$ If potentially atherogenic effects of CS are documented, are these mechanistically linked to calorigenesis? $(d)$ Does cessation of CS induce a rebound metabolic milieu that specifically favors accumulation of body fat (i.e., reduced fat mobilization or oxidation, increased fat synthesis) in the absence of altered food intake?

\section{Methods}

Human subjects. Volunteers were recruited by advertisement. Subjects gave written informed consent before enrollment and all protocols received prior approval from the University of California, San Francisco, Committee on Human Research. Seven heavy smokers (serum cotinine $>200 \mathrm{ng} / \mathrm{ml},>20$ cigarettes/d) were enrolled. Exclusion criteria were a history of medical illnesses (liver, kidney, metabolic, hematologic, pulmonary, gastrointestinal, neurologic), abnormalities on screening physical exam or by laboratory testing (chemistry profile, complete blood count), $>120 \%$ ideal body weight, or change in body weight $>10 \mathrm{lb}$ over the preceding $6 \mathrm{mo}$. Subjects were documented to be human immunodeficiency virus seronegative (since abnormalities of lipid metabolism may be present in asymptomatic seropositivity; reference 43). Subjects using medications with potential metabolic effects (e.g., $\beta$-blockers, $\beta$-agonists, theophylline, diuretics, glucocorticoids, phenytoin ) were also excluded. The seven subjects' age was $40 \pm 4$ yr, weight was $71.3 \pm 4.7 \mathrm{~kg}$, height was $172.3 \pm 3.1 \mathrm{~cm}$, and body mass index was $24.0 \pm 1.3 \mathrm{~kg} / \mathrm{m}^{2}$ (mean $\pm S E$ ). Duration of CS was $14 \pm 3 \mathrm{yr}$.

Study design. Subjects took part in a 2-wk inpatient metabolic ward study, 1 wk with CS and 1 wk without CS, in the General Clinical Research Center (GCRC) of San Francisco General Hospital. The sequence of CS and non-CS was randomized. Four subjects ended up in the CS phase first while three subjects were in the non-CS phase first. No effect of randomization sequence was observed here or previously (44). Subjects were placed on a constant, weight-maintaining metabolic diet throughout the GCRC admission, to avoid potential confounding effects of cessation of CS on food intake. Diets were prepared by the metabolic kitchen, after subjects and dieticians established a daily diet providing estimated energy needs (based on Harris-Benedict equations with comparison to 3-d dietary recalls for usual intake). Food intake was adjusted over the first $3 \mathrm{~d}$ on the metabolic ward on the basis of subjects' report of hunger, up to a maximum of $750 \mathrm{kcal} / \mathrm{d}$ above predicted energy needs.

On days 8 and 15 (day 7 of each phase), metabolic infusion studies were performed. These metabolic studies were designed to measure a number of metabolic processes: adipose lipolysis, reesterification of FFA in adipose and liver, de novo hepatic lipogenesis, cycling through fat oxidation/resynthesis, hepatic glucose production, fat oxidation, carbohydrate oxidation, and EE. The protocol consisted of intravenous infusions of sodium $\left[1-{ }^{13} \mathrm{C}\right]$ acetate $(0.090-0.120 \mathrm{mmol} / \mathrm{kg}$ per $\mathrm{h})$ from 2:00 a.m. until 6:00 p.m.; $\left[\mathrm{d}_{5}\right]$-glycerol $(9.7 \mu \mathrm{g} / \mathrm{kg}$ per $\mathrm{min})$ from 6:00 a.m. until noon; $\left[1,2,3,4-{ }^{13} \mathrm{C}\right]$ palmitate $(7 \mu \mathrm{g} / \mathrm{kg}$ per min $)$ complexed with human serum albumin $(25 \mathrm{~g})$ from 6:00 a.m. until noon; and [6,6-d2]-glucose $(0.04 \mathrm{mg} / \mathrm{kg}$ per $\mathrm{min})$ from 6:00 a.m. until noon. Indirect calorimetry was performed between 7:00 a.m. and 6:00 p.m. using a Deltatrac Metabolic Cart (Sensor Medix, Yorba Linda, CA) in the hooded mode. Urinary urea nitrogen was measured in order to calculate nonprotein respiratory quotient.

Subjects were fasted (noncaloric fluids allowed) from 10:00 p.m. of the preceding night until noon of the study day. Caffeine ingestion was not permitted during the metabolic study, and CS was not permitted between 10:00 p.m. and 9:00 a.m. Basal blood draws were performed every $10 \mathrm{~min}$ between 8:30 and 9:00 a.m. From 9:00 a.m. until noon, subjects smoked a cigarette every $30 \mathrm{~min}$ on the half hour and hour (CS phase) or did not smoke (non-CS phase). Repeat blood draws were every $10 \mathrm{~min}$ from 11:30 a.m. to noon. At noon, a meal of defined content was served, identical in both phases (consisting of 1,057 \pm 120 $\mathrm{kcal}, 42 \pm 6 \mathrm{~g}$ protein, $121 \pm 12 \mathrm{~g}$ carbohydrate, and $45 \pm 9 \mathrm{~g}$ fat), and smoking of one cigarette every $30 \mathrm{~min}$ continued until 2:00 p.m. (in the CS phase).

Compliance during the nonsmoking phase was established by measurement of breath carbon monoxide at random times throughout the day and urinary cotinine from $24-\mathrm{h}$ urine collections, as described previously (45). All subjects complained of nervousness, reduced concentration, and/or some irritability during the non-CS phase. No specific treatment for these or other withdrawal symptoms was instituted. Withdrawal scores were not measured, but we have found in previous studies that withdrawal scores are not high in a closed metabolic ward setting, due to the lack of external cues for smoking (Benowitz, N., unpublished observations).

Clinical laboratory measurements. Serum nicotine and cotinine concentrations were measured by gas chromatography (GC)/mass spectrometry (MS) (45). Serum lipids and thyroid function tests ( triiodothyronine $\left[\mathrm{T}_{3}\right]$, tetraiodothyronine $\left[\mathrm{T}_{4}\right]$ ) were measured by standard methods (Roche Laboratories, San Francisco, CA). Serum insulin was determined by radioimmunoassay. Serum FFA concentrations were measured by $\mathrm{GC} /$ flame ionization detection. Quantification was by comparison to known standards, with an internal standard of pentadecanoic acid added to the extraction mixture to determine recovery. Infusate glycerol concentrations were measured by glycerokinase enzyme assay, infusate glucose concentrations by glucose oxidase assay, and infusate palmitate concentrations by GC after extraction of the infusate with heptane/isopropyl alcohol (30:70). Urinary nitrogen was measured from 24-h collections by the Kjeldahl method.

Mass spectrometry. GC/MS (5971; Hewlett-Packard, Palo Alto, CA) was used for analysis of isotopic enrichments of serum glucose, glycerol, and fatty acid-methyl esters (FAME) from VLDL. A GC/MS ( 5970 ; HP) was used for analysis of FAME from serum. For glucosepentaacetate, we used a DB-17 60-m, 0.25-mm i.d. fused silica column. The molecular ion - acetate and its isotopomers (mass/charge $[\mathrm{m} / \mathrm{z}]$ 331 and 333) were quantified using selected ion monitoring (SIM) by comparison to standard curves of [6,6- $\left.\mathrm{d}_{2}\right]$-glucose at known enrichments. Chemical ionization was used. For FAME analyses, a 20-m fused DB-1 silica column, isothermal at $200^{\circ} \mathrm{C}$, was used with electron impact ionization. Ions at $\mathrm{m} / \mathrm{z} 270-274$ were monitored by SIM, representing the parent $M_{0}$ through the $M_{4}$ isotopomers with electron impact ionization. Glycerol triacetate was analyzed using a DB-225 column and chemical ionization, monitoring $\mathrm{m} / \mathrm{z} 159$ and 164 ( the molecular ion minus acetate, and its isotopomers). 
Statistical analyses. We performed two-factor repeated measures analysis of variance, with the two trial factors being smoking phase (chronic smoking vs. chronic nonsmoking) and time (baseline vs. 3-h values, the latter after acute smoking or nonsmoking). The existence of a significant chronic phase by time interaction represents statistical significance of acute smoking. If a significant interaction was found, follow-up comparisons of the four individual cell means were by Tukey's studentized range test at a procedurewise error rate of $5 \%$.

Calculations. The rate of appearance of glycerol ( $\mathrm{Ra}$ glycerol), RaFFA, and Ra glucose were calculated by the dilution technique (46):

$\mathrm{Ra}(\mu \mathrm{mol} / \mathrm{kg}$ per $\min )=[$ isotope infusion rate $(\mu \mathrm{mol} / \mathrm{kg}$ per $\mathrm{min}) /$

metabolite enrichment (M.E.)]-I,

where M.E. is molar excess, and $I$ is isotype infusion rate.

Reesterification of FFA, or lipolytic substrate cycling, was calculated using the method of Wolfe et al. and Klein et al. $(30,47)$. Reesterification of FFA can be intracellular in the adipocyte, or extracellular in the liver (Fig. 1). Reesterification in the adipocyte is calculated as the difference between $3 \times \mathrm{Ra}$ glycerol and RaFFA, based on the assumption that glycerol released from triglycerides must escape the adipocyte, due to the absence of glycerokinase (47), while FFA released from triglycerides can either be released or reesterified:

Intracellular recycling $(\mu \mathrm{mol} / \mathrm{kg}$ per $\mathrm{min})$

$=[3 \times \mathrm{Ra}$ glycerol $(\mu \mathrm{mol} / \mathrm{kg}$ per $\mathrm{min})]$

- RaFFA ( $\mu \mathrm{mol} / \mathrm{kg}$ per min).

Hepatic or extracellular reesterification is calculated as the difference between RaFFA and total body fat oxidation, representing nonoxidative FFA disposal. This calculation is based on the assumption that all FFA entering the circulation undergoes disposal by oxidation in tissues or reesterification in the liver and that fat oxidized in tissues at steady-state is derived from circulating FFA $(30,47)$ :

Hepatic reesterification $(\mu \mathrm{mol} / \mathrm{kg}$ per min)

$=\operatorname{RaFFA}(\mu \mathrm{mol} / \mathrm{kg}$ per min $)$

- fat oxidation ( $\mu \mathrm{mol} / \mathrm{kg}$ per $\mathrm{min})$.

RaFFA ( $\mu \mathrm{mol} / \mathrm{kg}$ per min $)$

$=$ Ra palmitate $(\mu \mathrm{mol} / \mathrm{kg}$ per $\mathrm{min}) /$

fraction of palmitate in FFA.

Fractional DNL was calculated using mass isotopomer distribution analysis, as described previously (48-50). The ratio of excess $\mathbf{M}_{2}$ /excess $M_{1}$ in FAME isotopomers from VLDL reveals the true precursor (acetyl-CoA) isotopic enrichment for de novo hepatic lipogenesis (DNL) during infusion of $\left[1-{ }^{13} \mathrm{C}\right]$ acetate, using probability analysis based on the binomial expansion $(48,51)$. The contribution from DNL to VLDL-palmitate is then calculated using the precursor-product relationship $(48,51)$ :

Fractional DNL $(\%)=100 \times\left[\mathrm{EM}_{1}(\right.$ M.E. $) / \mathrm{A}_{1}{ }^{*}$ (M.E.) $]$,

where $\mathrm{EM}_{1}$ is the enrichment of the M1 isotopomer of VLDL-palmitate, and $A_{1}{ }^{*}$ is the calculated asymptotic enrichment of the $M_{1}$ isotopomer if $100 \%$ of VLDL-palmitate were derived from hepatic DNL.

Absolute hepatic DNL was calculated by combining the calculations of fractional DNL with absolute hepatic reesterification of FFA (52). The fraction of VLDL-palmitate derived from DNL is known (eq. 5), so that the proportion from non-DNL (reesterification) is also known (unity minus fractional DNL). By combining this with the measured apparent hepatic reesterification rate, unidirectional or absolute hepatic DNL can be calculated whether NPRQ is $<1.0$ or $>1.0$ (53). When NPRQ is $<1.0$, the equation is:
Absolute hepatic DNL ( $\mathrm{mg} / \mathrm{kg}$ per min)

$=(\% \mathrm{DNL}) \times[\mathrm{RaFFA}-$ fat oxidation $(\mathrm{mg} / \mathrm{kg}$ per $\mathrm{min})]$.

This technique is based on several assumptions (53), including the assumption that fat oxidized in tissues derives from circulating FFA under steady-state fasting conditions. To the extent that tissues oxidize endogenous fat stores, which did not traverse the circulation, this technique may underestimate absolute DNL. The quantitative effect of deviations from this or other assumptions of the method can be calculated (53), and under the metabolic conditions present in these subjects, a relatively minor influence would be exerted even if a very large error due to oxidation of endogenous fat stores were introduced (see below).

The energy cost of lipolysis/reesterification substrate cycling was calculated as described elsewhere (30), assuming the ATP cost of triglyceride reesterification to be $8 \mathrm{~mol} \mathrm{ATP} / \mathrm{mol}$ triglyceride, and the energy value of ATP to be $20 \mathrm{kcal} / \mathrm{mol}$ ATP (54). The energy cost of $\mathrm{DNL} /$ fat oxidation cycling was calculated on the assumption that $28 \%$ of the energy content of carbohydrate is lost if it is converted to lipid before oxidation (54), or $3.2 \mathrm{kcal}$ lost $/ \mathrm{g}$ palmitate synthesized from glucose before oxidation.

\section{Results}

Serum nicotine, cotinine, and insulin concentrations. Basal serum nicotine concentrations after overnight abstention from CS during the smoking phase were $4.4 \pm 1.6 \mathrm{ng} / \mathrm{ml}$ (mean $\pm \mathrm{SE}$ ). Blood values during the $\mathrm{CS}$ period were drawn immediately before starting a cigarette (representing nadir concentrations) and rose to $33.2 \pm 4.8 \mathrm{ng} / \mathrm{ml}$ after $3 \mathrm{~h}$ of $\mathrm{CS}(P$ $<0.001)$, remaining increased $(26.7 \pm 0.8 \mathrm{ng} / \mathrm{ml} ; P<0.001$ vs. basal values) $2 \mathrm{~h}$ after lunch. During the non-CS week, basal levels were undetectable $(<1.0 \mathrm{ng} / \mathrm{ml})$. Basal serum cotinine concentrations in the smoking phase were $309 \pm 40 \mathrm{ng} / \mathrm{ml}$ and were not changed significantly by acute CS $(354 \pm 34 \mathrm{ng} / \mathrm{ml}$ after $3 \mathrm{~h}$ of $\mathrm{CS}$ and $347 \pm 53 \mathrm{ng} / \mathrm{ml} 2 \mathrm{~h}$ after lunch). Serum cotinine was $<10 \mathrm{ng} / \mathrm{ml}$ during the non-CS phase. Fasting serum insulin concentrations were not different between phases $(6.2 \pm 0.5 \mu \mathrm{U} / \mathrm{ml}$ in CS phase and $6.9 \pm 0.7 \mu \mathrm{U} / \mathrm{ml}$ in non-CS phase).

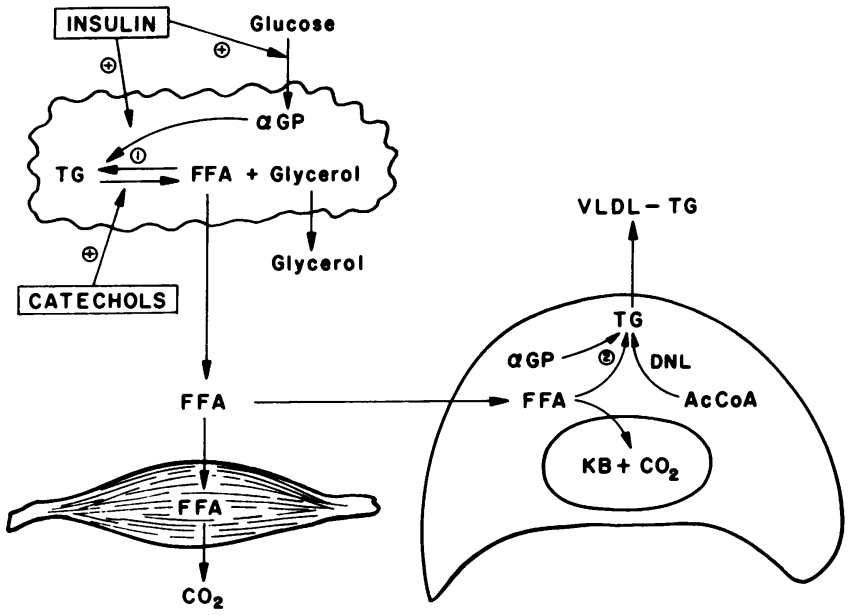

Figure 1. Sites of reesterification of FFA. 1, adipocyte; 2 , liver. $T G$, triglyceride; $\alpha G P, \alpha$-glycerol-phosphate; $D N L$, de novo lipogenesis; $A c C O A$, acetyl-CoA; $\mathrm{CO}_{2}$, carbon dioxide; $\mathrm{KB}$, ketone bodies; $\oplus$, stimulation of process indicated. 


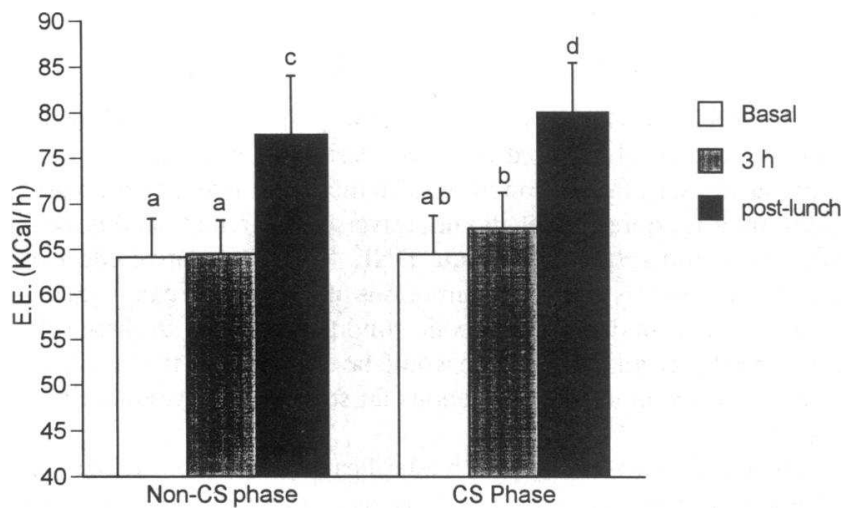

Figure 2. Effects of CS on resting EE. Details are described in the text. Categories sharing a common letter are not significantly different.

Energy expenditure. EE was measured hourly, during the 40-60th min of each hour. REE was not significantly different after an overnight fast in the CS and non-CS phases (64.4 \pm 3.5 and $64.1 \pm 4.3 \mathrm{kcal} / \mathrm{h}$, respectively; Fig. 2 ). REE increased by $4.6 \%$ after acute CS $(64.4 \pm 3.5$ to $67.3 \pm 3.9 \mathrm{kcal} / \mathrm{h})$ with increases observed in seven of seven subjects, but the change did not reach statistical significance when compared by repeated measures two-factor ANOVA to the analogous 3-h period of the non-CS phase $(P=0.18$ for phase by time interaction $)$. Ingestion of a mixed meal increased $\mathrm{EE}$ by $20.3 \%$ ( to $77.6 \pm 6.4$ $\mathrm{kcal} / \mathrm{h}$ ) in non-CS phase and by $18.9 \%$ (to $80.6 \pm 5.4 \mathrm{kcal} / \mathrm{h}$ ) in the CS phase $(P=0.82$ for phase by time interaction, $P<0.01$ for smoking phase vs. non-smoking phase all time points, and $P<0.002$ for postprandial vs. preprandial in each phase).

Serum FFA and lipid concentrations and lipolysis. FFA concentrations did not differ in the basal state between phases, but serum FFA concentrations increased from $381 \pm 94$ to $658 \pm 117 \mu \mathrm{M}$ after acute CS (a 73\% increase, $P<0.02$; Fig. 3 ). Serum FFA concentrations were unchanged $(228 \pm 36$ to $281 \pm 50 \mu \mathrm{M} ; \mathrm{NS}$ ) in the non-CS phase. RaFFA was not different after an overnight fast (basal state) in the CS and non-CS phases (Fig. 4). Acute CS significantly increased RaFFA by $77 \%$ (from $2.95 \pm 0.53$ to $5.21 \pm 0.72 \mu \mathrm{mol} / \mathrm{kg}$ per min compared with $2.43 \pm 0.24$ to $2.71 \pm 0.19 \mu \mathrm{mol} / \mathrm{kg}$ per min in nonCS phase $[P<0.001])$. Basal Ra glycerol was also not different

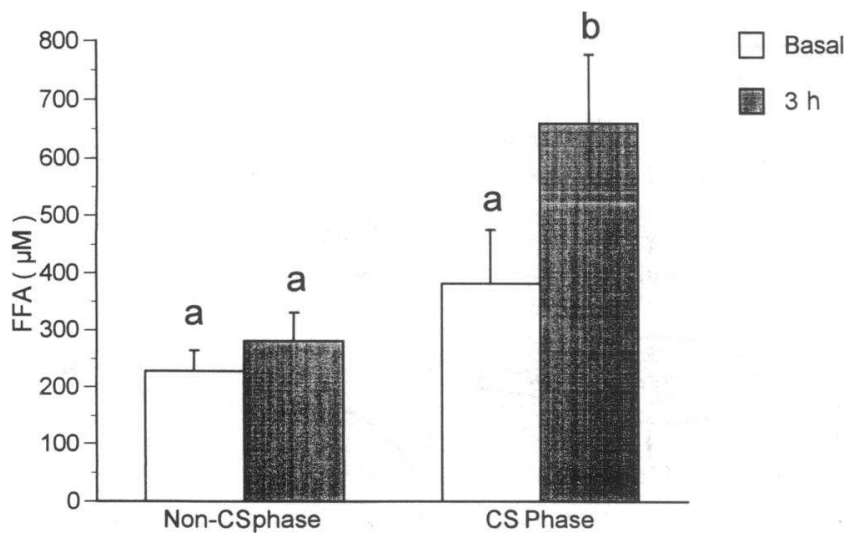

Figure 3. Effects of CS on serum FFA concentrations. Details are described in the text. Categories sharing a common letter are not significantly different.

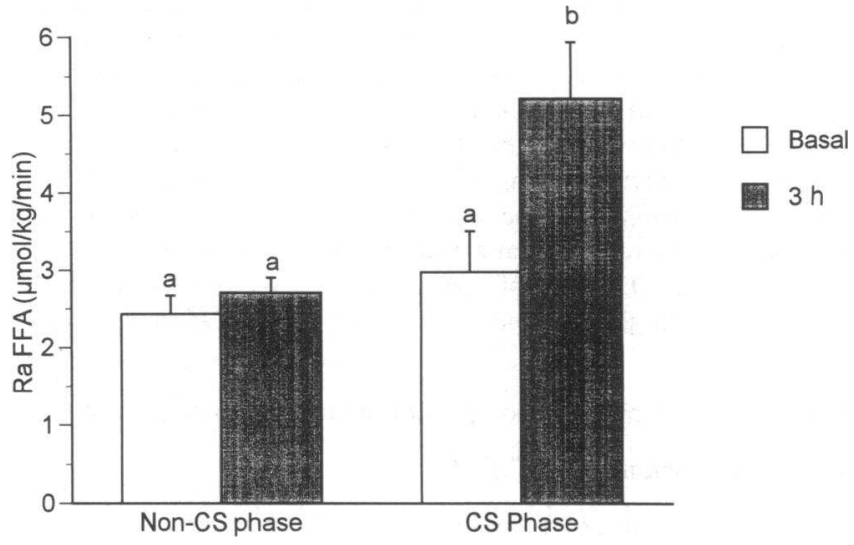

Figure 4. Effects of CS on Ra FFA. Details are described in the text. Categories sharing a common letter are not significantly different.

between CS and non-CS phases in the basal state (Fig. 5). Acute CS increased Ra glycerol by $82 \%$ from $1.15 \pm 0.20$ to $2.09 \pm 0.34 \mu \mathrm{mol} / \mathrm{kg}$ per min compared with $1.15 \pm 0.15$ to $1.08 \pm 0.10 \mu \mathrm{mol} / \mathrm{kg}$ per min in non-CS phase $(P<0.01)$. Basal serum lipid concentrations were not significantly different in the two phases (HDL cholesterol, $38 \pm 3$ and $41 \pm 3 \mathrm{mg} / \mathrm{dl}$ in CS and non-CS phases, respectively; triglycerides, $83 \pm 13$ and $96 \pm 17 \mathrm{mg} / \mathrm{dl}$ in CS and non-CS phases; total cholesterol, $165 \pm 28$ and $177 \pm 20 \mathrm{mg} / \mathrm{dl}$ in CS and non-CS phases; NS for all comparisons of CS and non-CS phases).

Substrate cycling of FFA and energy costs attributable to cycling. Intracellular (adipose) cycling of FFA increased by $0.57 \mu \mathrm{mol} / \mathrm{kg}$ per min after acute CS (Fig. 6), from $0.49 \pm 0.32$ to $1.06 \pm 0.58 \mu \mathrm{mol} / \mathrm{kg}$ per min, but the effect of acute CS did not achieve statistical significance $(P=0.10$ for phase by time interaction).

Extracellular (hepatic) cycling of FFA increased more than threefold, from $0.59 \pm 0.48$ to $2.03 \pm 0.54 \mu \mathrm{mol} / \mathrm{kg}$ per min after acute CS $(+1.44 \mu \mathrm{mol} / \mathrm{kg}$ per min; $P<0.03$; Fig. 7$)$. No change in hepatic reesterification of FFA was observed during the non-CS phase $(0.74 \pm 0.16$ to $0.53 \pm 0.23 \mu \mathrm{mol} / \mathrm{kg}$ per min, a change of $-0.21 \mu \mathrm{mol} / \mathrm{kg}$ per min; NS). The energy cost of lipolysis/reesterification of FFA, calculated as described above, is also shown (Table I). Energy costs of adipose cycling

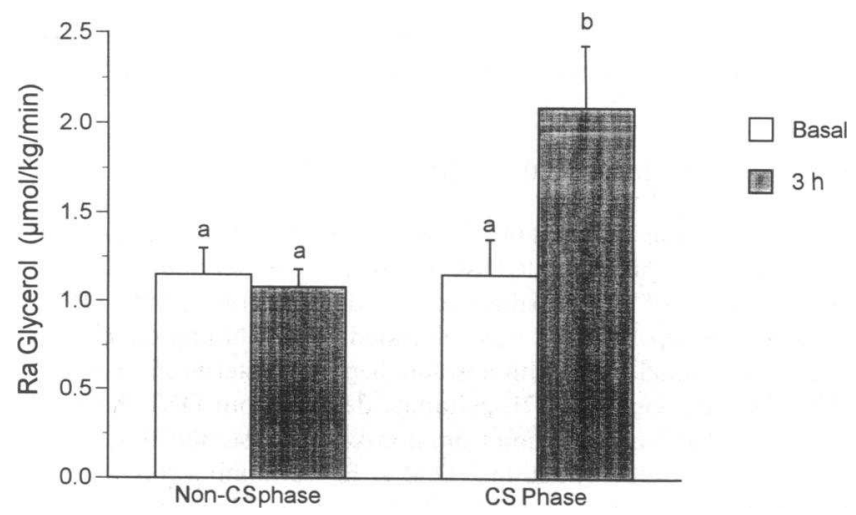

Figure 5. Effects of CS on Ra glycerol. Details are described in the text. Categories sharing a common letter are not significantly different. 


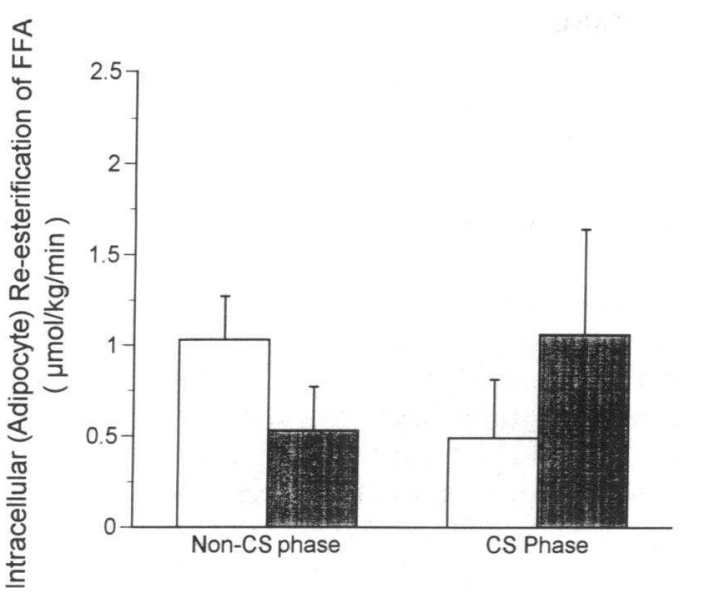

Figure 6. Effects of CS on adipoyte reesterification of FFA. Details are described in the text. No significant differences are present.

of FFA increased by $3.1 \mathrm{kcal} / 24 \mathrm{~h}$ (from 2.7 to $5.8 \mathrm{kcal} / 24 \mathrm{~h}$ ) after acute CS and decreased by $2.7 \mathrm{kcal} / 24 \mathrm{~h}$ in the non-CS phase. For hepatic cycling, the energy cost increased from 3.2 to $11.1 \mathrm{kcal} / \mathrm{d}(+7.9 \mathrm{kcal} / \mathrm{d})$ during the CS phase but did not increase from basal to 3-h measurements in the non-CS phase (from 4.1 to $2.9 \mathrm{kcal} / \mathrm{d}$ ).

$D N L$ and $D N L /$ fat oxidation substrate cycling. Fractional DNL was low and was unchanged by acute or chronic CS ( Fig. $8)$. Absolute DNL was estimated to be $0.5 \pm 0.2$ and $0.4 \pm 0.2$ $\mathrm{g} / \mathrm{d}$ at basal and 3-h time points in the non-CS phase and $0.4 \pm 0.2 \mathrm{~g} / \mathrm{d}$ in the basal state for CS phase, using the recently described method (53) that combines fractional DNL with hepatic reesterification of FFA. This technique underestimates absolute DNL to the extent that hepatic reesterification of FFA is underestimated. Since the latter depends on the assumption that intracellular fat oxidized had traversed the circulation in the form of FFA, oxidation of intracellular fat stores will result in an underestimation of absolute hepatic DNL (53). If we allow that intracellular fat provided $25 \%$ of total fat oxidation $(0.4-0.8 \mu \mathrm{mol} / \mathrm{kg}$ per $\mathrm{min})$, absolute hepatic DNL increases by $0.4 \mathrm{~g} / \mathrm{d}$. Even if we allow that intracellular fat provided as much substrate for oxidation as plasma FFA, absolute DNL only increases to $1.6-1.8 \mathrm{~g} / \mathrm{d}$. Thus, the low estimate of absolute hepatic DNL is not quantitatively sensitive to this deviation from the model under the metabolic conditions present here.

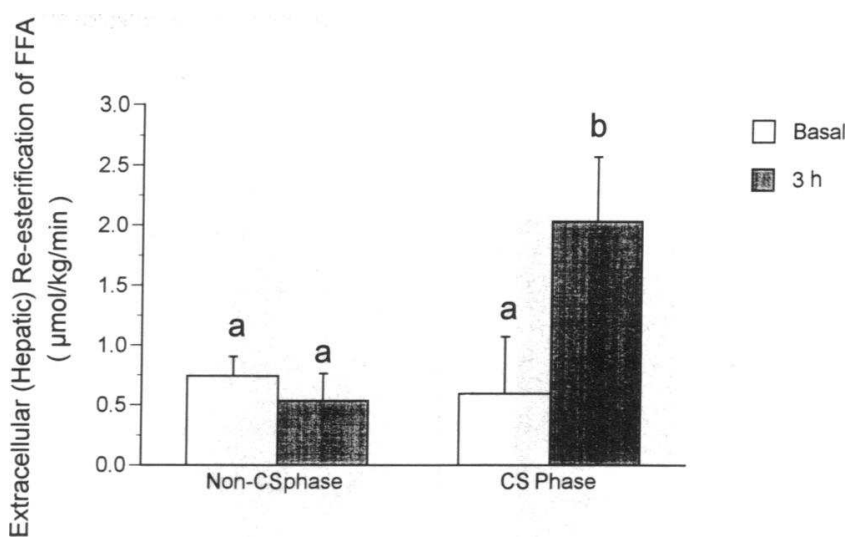

Figure 7. Effects of CS on hepatic reesterification of FFA. Details are described in the text. Categories sharing a common letter are not significantly different.

Ra glucose. Under fasting conditions, Ra glucose is equal to hepatic glucose production. Ra glucose values were higher after $3 \mathrm{~h}$ than $6 \mathrm{~h}$ of tracer infusion in both CS and non-CS phases (Fig. 9). This may represent the absence of an isotopic plateau after $3 \mathrm{~h}$ of tracer infusion. Alternatively, hepatic glucose production may have fallen during the additional $3 \mathrm{~h}$ of fasting. There was no effect of acute smoking on 6-h Ra glucose values $(2.08 \pm 0.21 \mathrm{mg} / \mathrm{kg}$ per $\mathrm{min}$ in CS phase vs. $1.67 \pm 0.13$ $\mathrm{mg} / \mathrm{kg}$ per $\mathrm{min}$ in non-CS phase; $P=0.28$ ).

Fuel selection. Nonprotein respiratory quotient (NPRQ) decreased from $0.88 \pm 0.02$ to $0.85 \pm 0.02$ during the 3 -h additional fasting period in the non-CS phase, and decreased from $0.83 \pm 0.02$ to $0.79 \pm 0.02$ in the CS phase ( significant time effect in both phases; $P<0.001$ ). There was no significant effect of acute smoking ( $P=0.73$ for phase by time interaction), although the NPRQ for all smoking phase time points was signifcantly lower than in the nonsmoking phase $(P<0.02)$. After lunch, NPRQ rose to $0.85 \pm 0.03$ and $0.85 \pm 0.01$ for CS and non-CS phases, respectively.

Whole body fat oxidation was significantly higher in the smoking phase than the nonsmoking phase $(2.36 \pm 0.33$ vs. $1.69 \pm 0.31 \mu \mathrm{mol} / \mathrm{kg}$ per min, respectively, at basal time point and $3.18 \pm 0.35$ vs. $2.19 \pm 0.24 \mu \mathrm{mol} / \mathrm{kg}$ per min at second time point; $P<0.02$ for CS vs. non-CS phase). Acute CS did not further increase whole body fat oxidation compared to non-CS phase $(P=0.42)$.

Table I. Lipid Substrate Cycling and Energy Costs

\begin{tabular}{|c|c|c|c|c|c|c|c|}
\hline \multirow[b]{2}{*}{ Study phase } & \multirow[b]{2}{*}{ Time } & \multicolumn{2}{|c|}{ Adipose cycling of FFA } & \multicolumn{2}{|c|}{ Hepatic cycling of FFA } & \multicolumn{2}{|c|}{ DNL/fat oxidation cycling } \\
\hline & & Rate & Energy cost & Rate & Energy cost & Rate & Energy cost \\
\hline & & umol/kg per min & $k c a l / d$ & $\mu \mathrm{mol} / \mathrm{kg}$ per min & $\mathrm{kcal} / \mathrm{d}$ & $g / d$ & $k c a l / d$ \\
\hline \multirow[t]{3}{*}{$\mathrm{CS}$} & Basal & $0.49 \pm 0.32$ & 2.7 & $0.59 \pm 0.48$ & 3.2 & $0.4 \pm 0.2$ & 1.3 \\
\hline & $3 \mathrm{~h}$ & $1.06 \pm 0.58^{*}$ & 5.8 & $2.03 \pm 0.54^{*}$ & 11.1 & ND & ND \\
\hline & Change & +0.57 & +3.1 & +1.44 & +7.9 & ND & ND \\
\hline \multirow[t]{3}{*}{ Non-CS } & Basal & $1.03 \pm 0.24$ & 5.6 & $0.74 \pm 0.16$ & 4.1 & $0.5 \pm 0.2$ & 1.6 \\
\hline & $3 \mathrm{~h}$ & $0.53 \pm 0.24$ & 2.9 & $0.53 \pm 0.23$ & 2.9 & $0.4 \pm 0.2$ & 1.3 \\
\hline & Change & $-0.49 \pm 0.21$ & -2.7 & -0.21 & -1.1 & -0.1 & -0.3 \\
\hline
\end{tabular}

See text for details of calculations. Energy costs of cycling are expressed as the costs if the measured rates were extrapolated over a 24-h period. ND, not done (steady-state not present for DNL measurement). ${ }^{*} P<0.05$ vs. basal values. 


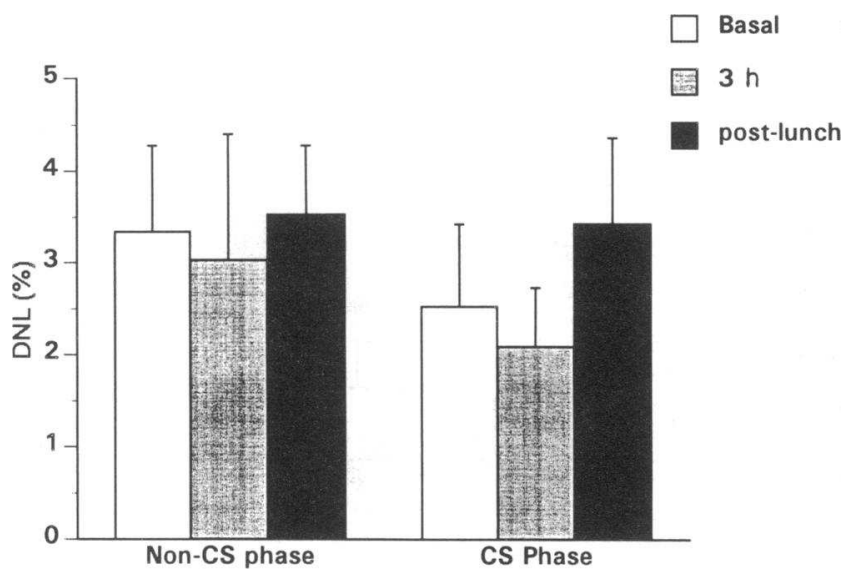

Figure 8. Effects of CS on DNL. Details are described in the text. No significant differences are present.

Whole body carbohydrate oxidation was nonsignificantly higher in the non-CS phase $(1.75 \pm 0.25$ vs. $1.28 \pm 0.21 \mathrm{mg} / \mathrm{kg}$ per min at basal time point and $1.48 \pm 0.17$ vs. $1.17 \pm 0.19 \mathrm{mg} /$ $\mathrm{kg}$ per min at second time point, for non-CS and CS phases, respectively; $P=0.06$ for comparison between phases). Acute CS did not significantly alter carbohydrate oxidation $(P=0.54$ vs. non-CS phase). After lunch, whole body carbohydrate oxidation increased in both phases $(2.09 \pm 0.41 \mathrm{mg} / \mathrm{kg}$ per min in CS phase and $1.92 \pm 0.31 \mathrm{mg} / \mathrm{kg}$ per min in non-CS phase; $P$ $<0.01$ for effect of lunch, NS for interaction between acute CS and lunch).

Dietary energy intake and body weight. Subjects ate $2,887 \pm 261 \mathrm{kcal} / \mathrm{d}$ in the CS phase and $3,092 \pm 302 \mathrm{kcal} / \mathrm{d}$ in the non-CS phase (NS). Predicted energy needs, based on the Harris-Benedict equation with an activity factor of 1.6 , were $2,714 \pm 163 \mathrm{kcal} / \mathrm{d}$.

Body weight increased by $0.5 \pm 0.1 \mathrm{~kg}$ in the CS phase ( $P$ $<0.05$ ) and $0.6 \pm 0.3 \mathrm{~kg}$ in the non-CS phase (NS between phases), consistent with the slightly positive energy balance estimated to be present.

\section{Discussion}

The major questions asked in this study were: $(a)$ What are the acute effects of CS on lipid metabolism, carbohydrate metabolism, and EE in heavy smokers? $(b)$ Are substrate cycles in-

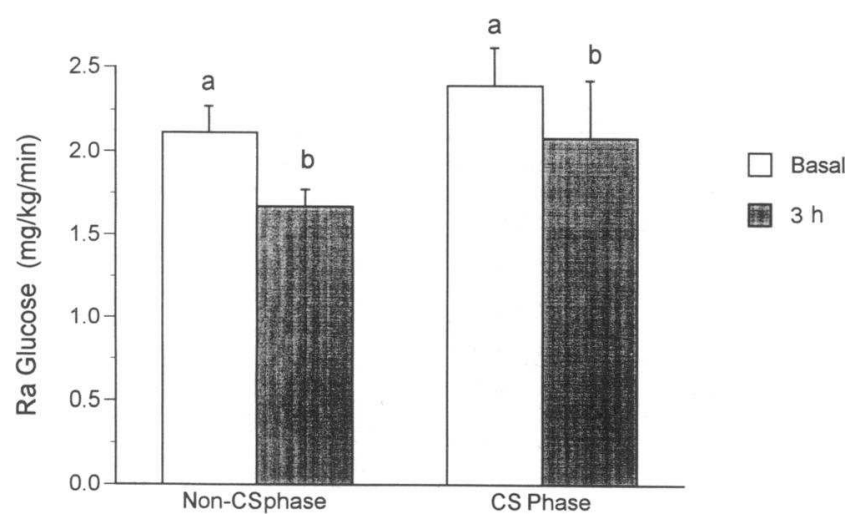

Figure 9. Effects of CS on Ra glucose. Details are described in the text. Categories sharing a common letter are not significantly different. duced by CS that could explain increased EE? (c) Are potentially atherogenic metabolic alterations induced by CS and, if so, are they integrally related to the thermogenic effects? And, $(d)$ are there rebound metabolic effects after cessation of CS that would specifically predispose to body fat accumulation in the absence of increased dietary energy intake?

We observed effects of acute CS on lipolysis, FFA substrate cycling, and REE but no effect on DNL, fat synthesis/fat oxidation substrate cycling, or hepatic glucose production. The lack of an effect on hepatic glucose production is interesting in view of the increased glycerol flux (a precursor for hepatic gluconeogenesis), increased FFA flux (a potential inhibitor of pyruvate oxidation and stimulator of gluconeogenesis, in accordance with the Randle cycle $[55,56])$, and increased catechol release $(23,29,57)$ induced by CS. Compensatory hepatic autoregulatory adjustments, such as reduced glycogenolysis (58) or slight increases in serum insulin, might have restrained hepatic glucose production in these normal subjects. Although CS-induced increases in circulating FFA concentrations had been demonstrated previously $(28,29)$, direct kinetic evidence that this is explicable by increased FFA entry rather than reduced FFA clearance in humans had not to our knowledge previously been reported. FFA concentrations increased proportionately to RaFFA ( 73 and $77 \%$, respectively), suggesting that the primary mechanism for elevated FFA concentrations is increased production. Since catechols increase FFA concentrations in humans (25-27) and nicotine releases catechols from the sympathetic nervous system $(23,58)$, mobilization of FFA is presumably mediated by catechols. The effect is not persistent, though, in that basal RaFFA, Ra glycerol, and FFA concentrations were not different during the CS and non-CS phases. The lack of a lipolytic stimulatory effect after overnight abstention from CS is also consistent with the pharmacology of nicotine (54). In contrast, the half-lives of serum lipoproteins are much longer (several days for LDL and HDL), so that effects induced by habitual CS during the course of the day might ultimately affect fasting lipoprotein concentrations $(32,33)$. Our 1-wk CS and non-CS periods were not long enough to allow differences in fasting serum lipoproteins to become apparent, however.

Alteration in lipolysis/reesterification substrate cycling were observed as a consequence of acute CS. Two types of substrate cycling can be distinguished, intracellular (adipocyte) reesterification and extracellular (hepatic) reesterification $(30,47)$. Measurement of Ra glycerol represents absolute lipolysis, since the adipocyte lacks glycerokinase needed to reuse glycerol released from triglyceride (47), whereas RaFFA reflects FFA that escaped reuse within the adipocyte (Fig. 1). The difference between $3 \times \mathrm{Ra}$ glycerol and RaFFA therefore is an index of intracellular reesterification of FFA. We observed a nonsignificant $(P=0.10)$ increase in intracellular reesterification during acute CS (Fig. 6). Intracellular cycling represents a thermogenic process that is relatively benign in terms of systemic metabolism, the only systemic consequence being release of glycerol. In contrast, extracellular recycling also involves entry of FFA into the systemic circulation and reesterification in the liver, which will tend to increase hepatic VLDL-triglyceride (TG) production and secondarily alter plasma lipoproteins (35-41).

Of the two possible sites for reesterification of FFA, the liver is clearly the least desirable metabolically. Unfortunately, this process is significantly stimulated by acute CS (Fig. 7). The 
increase in extracellular reesterification of FFA represents a mechanism by which CS might increase VLDL-TG production and alter serum lipids in an atherogenic direction (35-37). The association of increased FFA flux and delivery to the liver with high cardiovascular risk (e.g., diabetes [35]; CS), or reduced FFA flux with reduced cardiovascular risk (e.g., nicotinic acid therapy [38-40]), raises the possibility that this is a general causal relationship. It will be of interest to evaluate how these lipid metabolic effects of CS are influenced by the presence of other cardiovascular risk factors, such as hypertension, diabetes, insulin resistance, and obesity. Systemic FFA release may represent a mechanistic link between CS or nicotine and other risk factors. If so, the benefit of nicotine replacement therapy to prevent weight gain after cessation of CS $(59,60)$ may have to be considered in this light. The effect of nicotine replacement therapy (i.e., nicotine patches and chewing gum) on energy expenditure, lipid kinetics, and serum lipoproteins is therefore an important area for future research.

To the extent that CS-induced thermogenesis is mediated by substrate cycling of FFA, the sought-after thermogenic effect and the undesirable, possibly atherogenic effect on lipids might be two sides of the same coin, i.e., inseparable because they are based on a shared lipolytic mechanism. Our quantitative calculations do not support this notion, however. The total thermogenic contribution from reesterification of FFA was calculated to be only $11.0 \mathrm{kcal} / \mathrm{d}$, or $15 \%$ of observed changes in REE after acute CS in these heavy smokers. The implication is that inhibition of lipolysis and subsequent reesterification might substantially reduce dyslipidemic effects but not prevent any thermogenic effects of CS, if these results in heavy smokers also apply in light smokers, in whom CS-induced thermogenesis is greater (19-22). This mechanistic question is not just of theoretical interest. Although it certainly would not be desirable from a general health point of view to administer a lipolysis inhibitor in order to allow people to continue smoking with less atherogenic risk, the question may have practical relevance for use of nicotine patches. Niacin is believed to increase adipocyte reesterification of FFA and thereby reduce FFA release (38). If this mechanism of action of niacin can be confirmed, it might in principle maintain the net reesterification rate induced by nicotine but redirect it to the adipocyte and away from the liver. We are in the process of determining the effect of nicotine patch therapy on EE and lipid metabolism.

The thermogenic effect of acute CS in these heavy smokers ( $\sim 5 \%$ and not statistically significant) was less than that reported by some previous investigators. Hofstetter et al. (19) reported an $8 \%$ increase in 24-h expenditure. Glauser et al. (33) found that cessation of CS caused an $8 \%$ decrease in EE. On the other hand, Perkins et al. (20) reported that intranasal nicotine $(15 \mu \mathrm{g} / \mathrm{kg})$, which achieves serum nicotine concentrations similar to those observed in our subjects during CS, increased EE by $\sim 6 \%$. We have elsewhere observed (22) that intravenous nicotine administration has a lower thermogenic effect in heavy smokers than light smokers, which may explain some of the differences between studies. Nevertheless, heavy smokers continue to be exposed to the lipolytic and hepatic FFA reesterifying effects of CS even while experiencing less thermogenesis than light smokers.

Finally, we asked whether rebound effects on REE or intermediary metabolism that would specifically promote fat deposition would be apparent after 1 wk of non-CS. Basal fat mobilization was not reduced nor was new fat synthesis (de novo lipogenesis) increased by cessation of CS. REE was also unchanged. Thus, in the absence of surplus energy intake, the only metabolic driving force that could promote gain of body fat after cessation of CS was reduced whole body fat oxidation. Carney and Goldberg (41) reported an increase in serum lipoprotein lipase (LPL) activity after cessation of CS, and suggested that a predisposition to accrual of body fat would result. It should be pointed out that, on metabolic principles, increased flux of triglyceride through LPL would not result if there were increased LPL activity, since there is no alternative fate for serum triglyceride. Rather, a lower serum triglyceride concentration with the same flux would result, at steady-state. Increased flux through LPL has not been documented after cessation of CS. Accordingly, we do not believe that there currently exists convincing evidence for a rebound metabolic milieu that specifically favors body fat gain after cessation of CS. Through its acute calorigenic effects, CS may induce a lower body weight than would otherwise be present in an individual, but the proposal that cessation of CS should result in an overshoot (higher than usual) body weight or body fat content due to increased fat synthesis or storage remains to be proven quantitatively, in the absence of overeating. Our results also provide no purely metabolic rationale for provision of nicotine to prevent fat gain after cessation of CS, independent of effects on food intake or thermogenesis.

In conclusion, acute CS in heavy smokers results in increased FFA entry into the circulation, elevated serum FFA concentrations, and increased hepatic reesterification of FFA, which may explain the dyslipidemic effect of CS. Although hepatic reesterification represents a substrate cycle, it contributes only a small proportion of any calorigenesis induced by acute CS, implying that the thermogenic and potentially atherogenic effects of nicotine may not be integrally connected. Thus, heavy smokers are exposed to deleterious alterations in lipid metabolism despite a reduced thermogenic effect and can stop smoking without fear of inducing a rebound metabolic propensity favoring fat gain in the absence of increased food intake.

\section{Acknowledgments}

We gratefully acknowledge the technical help of Irving Fong, the nurses in the San Francisco General Hospital GCRC for their help, and Cici Hyde for typing of the manuscript.

This work was supported by grant 1 RT 475 from the Tobacco-Related Disease Research Program of the University of California (to M. K. Hellerstein); grant DA-2277 from the National Institutes of Health (to N. L. Benowitz), and the Division of Research Resources (NIH grant RR-00083) for the General Clinical Research Center. J. M. Schwarz was supported by the Fonds National Suisse de la Recherche Scientifique and by Fondation Raymond Berger.

\section{References}

1. Gritz, E. R., R. C. Klesges, and A. W. Meyers. 1989. Smoking and body weight: implications for interventions and post-cessation weight control. Ann. Behav. Med. 11:144-153.

2. Rigotti, N. A. 1989. Cigarette smoking and body weight. N. Engl. J. Med. 320:931-933.

3. Pirie, P. L., D. M. Murray, and R. V. Luepher. 1991. Gender differences in cigarette smoking and quitting in a cohort of young adults. Am. J. Public Health 81:324-327.

4. Weekley, C. K. III, R. C. Klesges, and G. Reglen. 1992. Smoking as a weight control strategy and its relation to smoking status. Addict. Behav. 17:259-271. 
5. Grunberg, N. E. 1986. Behavioral and biological factors in the relationship between tobacco use and body weight. In Advances in Behavioral Medicine. Vol. 2. E. S. Katkin and S. B. Manuck, editors. Jai Press Inc., Greenwich, Cc. 97-129. 6. Wack, J. T., and J. Rodin. 1982. Smoking and its effects on body weight and systems of caloric regulation. Am. J. Clin. Nutr. 35:366-380.

7. Comstock, G. W., and R. W. Stone. 1972. Changes in body weight and subcutaneous fatness related to smoking habits. Arch. Environ. Health. 24:271276.

8. Williamson, D. F., J. Madans, R. F. Anda, J. C. Kleinman, G. A. Giovino, and T. Byers. 1991. Smoking cessation and severity of weight gain in a national cohort. N. Engl. J. Med. 324:739-745.

9. Hall, S. M., D. Ginsberg, and R. T. Jones. 1986. Smoking cessation and weight gain. J. Consult. Clin. Psychol. 54:342-346.

10. Department of Health and Human Services. 1990. Health Benefits of Smoking Cessation. A Report to the Surgeon General. Government Printing Office, Washington, DC. Department of Health and Human Services publication no. (CDC) 90-8416.

11. Gordon, R., W. B. Kannel, T. R. Dawber, and D. McGee. 1978. Changes associated with quitting cigarette smoking: the Framingham Study. Am. Heart J. 90:322-328.

12. Department of Health and Human Services, Public Health Service. 1989. The Health Consequences of Smoking: 25 Years of Progress. A Report of the Surgeon General. Government Printing Office, Washington, DC. Department of Health and Human Services publication no. (CDC) 89-8411.

13. Hebek, K. A., N. E. Miller, and O. D. Mjos. 1975. Effects of nicotine and inhalation of cigarette smoke on total body oxygen consumption in dogs. Scand. J. Clin. Lab. Invest. 35:67-72.

14. Blackburn, H. I., J. Brozeck, and H. L. Taylor. 1960. Common circulatory measurements in smokers and nonsmokers. Circulation. 22:1112-1124.

15. Rodin, J. 1987. Weight changes following smoking cessation: the role of food intake and exercise. Addict. Behav. 12:303-317.

16. Stamford, B. A., S. Matter, R. D. Fell, and P. Papanek. 1986. Effects of smoking cessation on weight gain, metabolic rate, caloric consumption and blood lipids. Am. J. Clin. Nutr. 43:486-494.

7. Jacobs, M. A., P. H. Knapp, L. S. Anderson, N. Karush, R. Meissner, and S. J. Richman. 1968. Relationship of oral frustration factors with heavy cigarette smoking in males. J. Nerv. Ment. Dis. 141:161-171.

18. Schecter, M. D., and P. G. Cook. 1976. Nicotine-induced weight loss in rats without an effect on appetite. Eur. J. Pharmacol. 38:63-69.

19. Hofstetter, A., Y. Schutz, E. Jequier, and J. Wahren. 1986. Increased 24-hour energy expenditure in cigarette smokers. N. Engl. J. Med. 314:79-82.

20. Perkins, K. A., L. H. Epstein, B. L. Marks, R. L. Stiller, and R. G. Jacob. 1989. The effect of nicotine on energy expenditure during light physical activity. N. Engl. J. Med. 320:898-903.

21. Perkins, K. A., L. H. Epstein, R. L. Stiller, J. E. Sexton, M. H. Fernstrom, R. G. Jacob, and R. Solverg. 1990. Metabolic effects of nicotine after consumption of a meal in smokers and non-smokers. Am. J. Clin. Nutr. 52:228-233.

22. Arcavi, L., and N. L. Benowitz. 1992. Differential tolerance to metabolic and cardiovascular effects of nicotine. Clin. Pharmacol. \& Ther. 51:170A

23. Watts, D. T. 1960. The effect of nicotine and smoking on the secretion of epinephrine. Ann. NY Acad. Sci. 90:74-80.

24. Ellis, S. 1956. The metabolic effects of epinephrine and related amines Pharmacol. Rev. 8:485.

25. Havel, R. J., L. A. Carlson, L. Ekelund, and A. Holmgren. 1964. Studies on the relation between mobilization of free fatty acids and energy metabolism in man: effects of norepinephrine and nicotinic acid. Metabolism. 13:1402-1412.

26. Steinberg, D., P. J. Nestel, R. Elsworth, R. Buskirk, and R. H. Thompson 1964. Calorigenic effect of norepinephrine correlated with plasma free fatty acid turnover and oxidation. J. Clin. Invest. 43:167-176.

27. Frederickson, D. S., and R. S. Gordon, Jr. 1956. Transport of fatty acids. Physiol. Rev. 8:485.

28. Bizzi, A., M. T. Tacconi, A. Medea, and S. Garattini. 1972. Some aspects of the effect of nicotine on plasma FFA and tissue triglycerides. Pharmacology (Basel). 7:216-224.

29. Kershbaum, A., R. Khorsandian, R. F. Caplan, S. Bellet, and L. J. Feinberg. 1963. The role of catecholamines in the free fatty acid response to cigarette smoking. Circulation. 28:52-57.

30. Wolfe, R. R., D. N. Herndon, F. Jahoor, H. Miyoshi, and M. Wolfe. 1987 Effect of severe burn injury on substrate cycling by glucose and fatty acids. $N$ Engl. J. Med. 317:403-408.

31. Mjos, O. D. 1988. Lipid effects of smoking. Am. Heart J. 115:272-275.

32. Billimoria, J. D., H. Pozner, B. Metselaar, F. W. Best, and D. C. James. 1975. Effects of cigarette smoking on lipids, lipoproteins, blood coagulation, fibrinolysis, and cellular components of human blood. Arteriosclerosis. 21:61-76.

33. Glauser, S. C., E. M. Glauser, M. M. Reidenberg, B. F. Rusy, and R. J. Tallarida. 1970. Metabolic changes associated with the cessation of cigarette smoking. Arch. Environ. Health. 20:377-381.

34. Muscat, J. E., R. E. Harris, N. J. Haley, and E. L. Wynder. 1991. Cigarette smoking and plasma cholesterol. Am. Heart J. 121:141-147.
35. Reaven, G., and M. Greenfield. 1981. Evidence for three clinical syndromes. Diabetes. 30(Suppl. 2):66-75.

36. Stern, M. D., and S. M. Haffner. 1991. Dyslipidemia in type II diabetes. Implications for therapeutic intervention. Diabetes Care. 14:1144-1159.

37. Reaven, G. M., H. Chang, and B. B. Hoffman. 1988. Additive hypoglycemic effects of drugs that modify free-fatty acid metabolism by different mechanisms in rats with streptozotocin-induced diabetes. Diabetes. 37:28-33.

38. Figge, H. L., J. Figge, P. F. Souney, A. H. Mutnick, and F. Sacks. 1988 Nicotinic acid: a review of its clinical use in the treatment of lipid disorders. Pharmacotherapy. 8:287-294.

39. Carlson, L. A., and L. Oro. 1962. The effect of nicotinic acid on the plasma free fatty acids. Demonstration of a metabolic type of sympathicolysis. Acta Med. Scand. 172:641.

40. Carlson, L. A., R. J. Havel, L.-G. Ekelund, and A. Holmgrem. 1963 Effect of nicotinic acid on the turnover rate and oxidation of the free fatty acids of plasma in man during exercise. Metabolism. 12:837.

41. Carney, R. M., and A. P. Goldberg. 1984. Weight gain after cessation of cigarette smoking: a possible role of adipose-tissue lipoprotein lipase. $N$. Engl. J. Med. 310:614-616.

42. Chajek-Shaul, T., E. M. Berry, E. Ziv, G. Friedman, O. Stein, G. Scherer, and Y.Stein. 1990. Smoking depresses adipose lipoprotein lipase response to oral glucose. Eur. J. Clin. Invest. 20:299-304.

43. Hellerstein, M. K., C. Grunfeld, K. Wu, M. Christiansen, S. Kaempfer, C. Kletke, and C. H. L. Shackleton. 1993. Increased de novo hepatic lipogenesis in human immunodeficiency virus infection. J. Clin. Endocrinol. \& Metab. 76:559 565.

44. Neese, R. A., N. L. Benowitz, R. Hoh, D. Faix, I. Fong, A. Basinger, K Pun, and M. K. Hellerstein. 1993. Effects of surplus dietary energy intake on lipogenesis, lipolysis, fat oxidation and substrate cycling in humans during cigarette smoking and after its cessation. Clin. Res. 41:298A. (Abstr.)

45. Jacob, P., L. Yu, M. Wilson, and N. L. Benowitz. 1991. Selected ion monitoring for determination of nicotine, cotinine, and deuterium-labeled analogs: absence of an isotope effect in the clearance of (S)-nicotine-3', 3'- $\mathrm{D}_{2}$ in humans. Biol. Mass Spectrom. 20:247-252.

46. Wolfe, R. R. 1984. Tracers in Metabolic Research. Radio-Isotope and Stable Isotope/Mass Spectrometric Methods. Alan R. Liss, Inc., New York. 471 pp.

47. Klein, S., V. R. Young, G. L. A. Blackburn, B. R. Bistrian, and R. R. Wolfe. 1986. Palmitate and glycerol kinetics during brief starvation in norma weight young adult and elderly subjects. J. Clin. Invest. 78:928-933.

48. Hellerstein, M. K., M. Christiansen, S. Kaempfer, C. Kletke, K. Wu, J. S. Reid, N. S. Hellerstein, and C. H. L. Shackleton. 1991. Measurement of de novo hepatic lipogenesis in humans using stable isotopes. J. Clin. Invest. 87:18411852.

49. Hellerstein, M. K., K. Wu, S. Kaempfer, C. Kletke, and C. H. L. Shackleton. 1991. Sampling the lipogenic hepatic acetyl-CoA pool in vivo in the rat Comparison of xenobiotic probe to values predicted from isotopomeric distribution in circulating lipids and measurement of lipogenesis and acetyl-CoA dilution. J. Biol. Chem. 266:10912-10919.

50. Park, O.-J., D. Cesar, D. Faix, K. Wu, C. H. L. Shackleton, and M. K. Hellerstein. 1992. Mechanisms of fructose-induced hypertriglyceridemia in the rat: activation of hepatic pyruvate dehydrogenase (PDH) through inhibition of PDH kinase. Biochem. J. 282:753-757.

51. Hellerstein, M. K., and R. Neese. 1992. Mass isotopomer distribution analysis: a technique for measuring biosynthesis and turnover of polymers. $\mathrm{Am}$. J Physiol. 263:E988-E1001.

52. Hellerstein, M. K., R. A. Neese, and J.-M. Schwarz. 1993. Measurement of absolute rates of hepatic de novo lipogenesis and re-esterification of free fatty acids in humans. FASEB (Fed. Am. Soc. Exp. Biol.) J. 7:A280. (Abstr.)

53. Hellerstein, M. K., R. A. Neese, and J.-M. Schwarz. 1993. Measurement of absolute rates of hepatic de novo lipogenesis and re-esterification of free fatty acids in humans. Am. J. Physiol. In press.

54. Flatt, J. P. 1978. The biochemistry of energy expenditure. Recent Adv Obes. Res. 2:211-217.

55. Randle, P. J., C. N. Hales, P. B. Garland, and E. A. Newsholme. 1963. The glucose fatty-acid cycle. Its role in insulin sensitivity and the metabolic disturbances of diabetes mellitus. Lancet. i:7285-7289.

56. Randle, P. J. 1986. Fuel selection in animals. Biochem. Soc. Trans. 14:799-806.

57. Benowitz, N. L. 1988. Pharmacologic aspects of cigarette smoking and nicotine addiction. $N$. Engl. J. Med. 319:1318-1330.

58. Giaccari, A., and L. Rossetti. 1992. Predominant role of gluconeogenesis in the hepatic glycogen repletion of diabetic rats. J. Clin. Invest. 89:36-45.

59. Hajek, P., P. Jackson, and M. Belcher. 1988. Long-term use of nicotine chewing gum: occurence, determinants and effect on weight gain. J. Am. Med. Assoc. 260:1593-1596.

60. Sutherland, G., J. A. Stapleton, M. A. Russell, M. J. Jarvis, P. Harek, M Belcher, and C. Feyerabend. 1992. Randomized controlled trial of nasal nicotine spray in smoking cessation. Lancet. 340:324-329. 